\title{
Retinoblastoma metastatic to the contralateral orbit
}

\author{
Kanwar Mohan, Amod Gupta, J S Saini, Subhadra Jalali, Kusum Joshi
}

\begin{abstract}
In a 9-month-old girl a subperiosteal metastasis of the maxillary bone developed in the contralateral orbit about one year after enucleation of a poorly differentiated retinoblastoma.
\end{abstract}

Studies of necropsy cases of retinoblastoma have shown that about $94-100 \%$ of patients have some form of intracranial metastasis and $53 \%$ have involvement of distant bones. ${ }^{1-3}$ The involvement of orbital bones is by direct spread from the recurrent tumour in the affected orbit, though some authors have considered ipsilateral orbital involvement to be orbital metastases. ${ }^{1-3}$ To our knowledge there is no report of retinoblastoma metastasising to the bones of the contralateral orbit. We report an unusual case of unilateral retinoblastoma in which the patient developed subperiosteal metastasis in the contralateral maxillary bone.

\section{Case history}

A 9-month-old girl had had a white pupillary reflex in her right eye since the age of 3 months. Her right eye did not fix light. The anterior segment in this eye appeared normal. On ophthalmoscopy a large greyish-white endophytic mass with prominent feeder vessels was seen in the temporal half of the retina. The intraocular pressure in this eye was normal. Her left eye was normal. A clinical diagnosis of retinoblastoma in the right eye was made and enucleation was advised.

One week later, the child developed orbital inflammation, with swelling of lids, conjunctival chemosis, proptosis, and restricted motility of the right eye. She was afebrile. She responded well to oral anti-inflammatory drugs but was lost to follow up.

When she was seen again 16 months later the anterior segment in her right eye showed signs of healed iritis but no appreciable difference in the morphology of the retinal mass. The intraocular pressure was low, and there were no signs of orbital involvement. Her left eye and orbit were normal. Systemic examination did not reveal any signs of distant metastases. The right eye was enucleated and 800 rads of external radiation were delivered to the orbit. Histopathological examination of the enucleated eye showed poorly differentiated retinoblastoma with extensive necrosis (Fig 1). The iris and ciliary body were necrotic, with focal invasion of the choroid. The optic nerve was free of the tumour.

Eleven months after enucleation of the right eye she developed fullness in the lateral part of the left lower eyelid (Fig 2). The left eyeball was normal but displaced upwards. Eyeball movements were normal. There were no signs of tumour recurrence in the right orbit or of

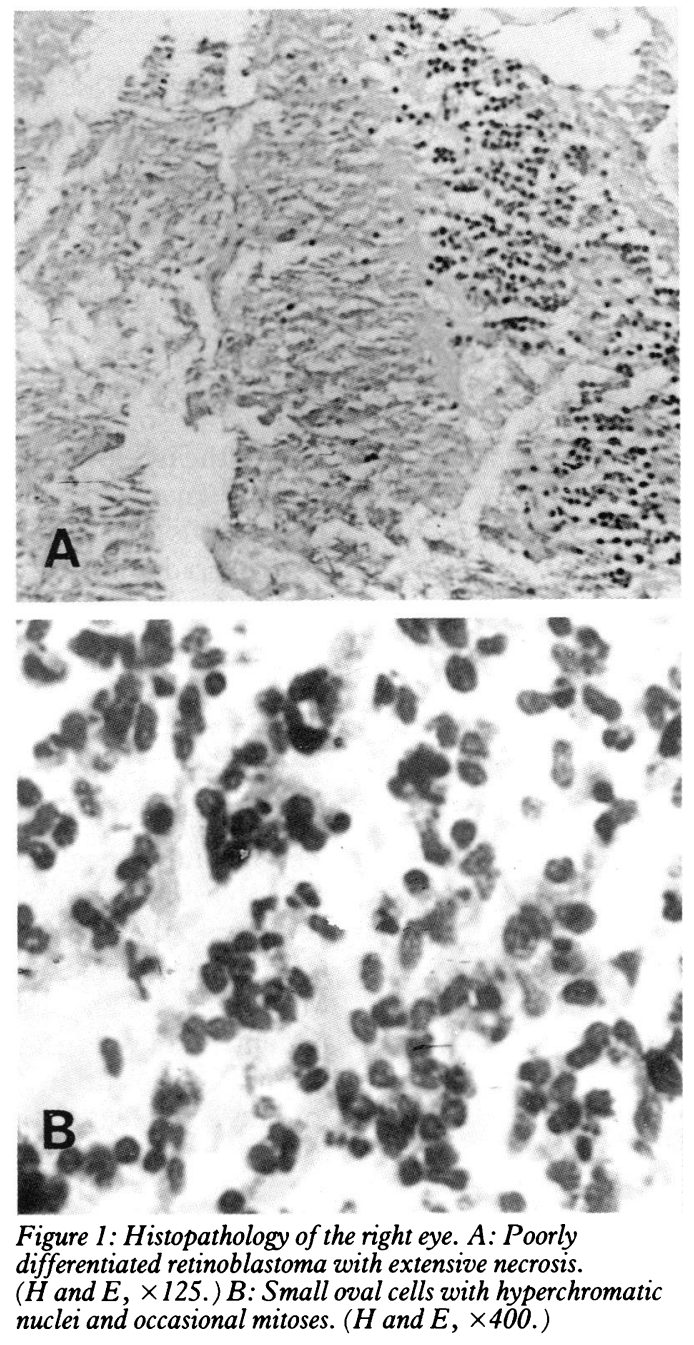

intracranial or distant metastases. A fine needle aspiration biopsy from the mass was consistent with metastatic retinoblastoma. The orbital mass was treated with 800 rads of external radiation and oral cyclophosphamide, but showed no response. On surgical exploration the mass was found to be subperiosteal. A wedge biopsy showed a histopathological picture identical to that of the primary tumour. Two weeks later the



Figure 2: Fullness of the outer part of the left lower eyelid 11 months after enucleation of the right eye for retinoblastoma. Note slight upward displacement of the left eyeball. 


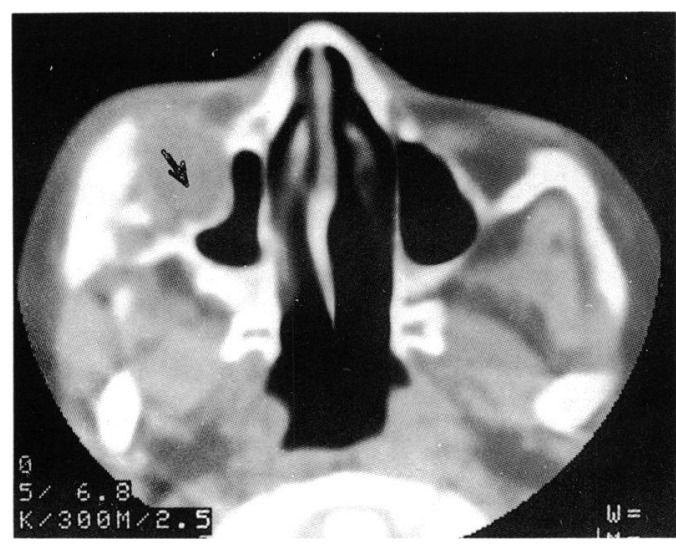

Figure 3: Axial CT scan shows the mass indenting the roof of the left maxillary sinus (arrow) but without invasion.

mass increased rapidly and an axial CT scan revealed a soft tissue mass in the anterolateral part of the left lower orbit, with mass impression over the maxillary sinus without actual invasion of the sinus (Fig 3). Treatment with 1800 rads of external radiation led to an initial regression, but within six weeks the tumour invaded the left maxillary sinus and infratemporal fossa. The child died one year after the first appearance of the metastasis.

\section{Discussion}

The distant bones most commonly affected by retinoblastoma metastasis are the ribs and the vertebrae, these being the most active in the haemopoietic system, especially in children.' Metastasis to the contralateral orbit in our patient is unique. (Shields $\mathrm{J} \mathrm{A}$, personal communication). The metastasis was subperiosteal initially, but later the underlying bone was eroded and the tumour grew into the maxillary antrum. Most of the cases with metastases to the distant bones have involvement of the skull bones as well as the viscera. ${ }^{3}$ Involvement of these structures cannot be entirely ruled out in our patient, since laboratory investigations often fail to detect metastases. ${ }^{3}$ A non-ocular tumour was ruled out by an identical histological appearance in our patient ${ }^{+}$(Shields $\mathrm{J} \mathrm{A}$, personal communication). The average time lag between initial diagnosis of retinoblastoma and the first signs and symptoms of metastasis varies from $5 \cdot 5$ months to $2 \cdot 1$ years. ${ }^{1-3}$ In our patient the time interval was $2 \cdot 3$ years.

Patients with tumour recurrence in the orbit are more likely to have distant metastases. ' Our patient did not show ipsilateral orbital recurrence at any stage of the disease. Choroidal invasion and a poorly differentiated histological type were the only apparent risk factors. ${ }^{5-8} \mathrm{We}$ believe that 16 months delay in enucleation perhaps allowed dissemination of the tumour cells. Once metastases appear, the average survival time is 3.5 to 5.8 months $^{23}$ (Shields J A personal communication). Early diagnosis and prompt treatment are of prime importance in reducing the mortality from retinoblastoma.

We are grateful to the staff of the Armed Forces Institute of Pathology, Washington, DC, For histopathological examination of the biopsy from the left orbital mass.

1 Carabajal UM. Metastasis in retinoblastoma. Am $\mathcal{f}$ Ophthalmol 959; 48: 47-69.

Merriam GR. Retrinoblastoma: analysis of 17 autopsies. Arch Ophthalmol 1950; 44: 71-108.

3 MacKay J, Abramson DH, Ellsworth RM. Metastatic patterns of retinoblastoma. Arch Ophthalmol 1984; 102: 391-6.

4 Abramson DH, Ellsworth RM, Zimmerman LE. Nonocular cancer in retinoblastoma survivors. Ophthalmology 1976; 81: $454-7$.

5 Zimmerman LE. The registry of ophthalmic pathology: past, present and future. Ophthalmology 1961; 65: 51-113.

6 Brown DH. The clinicopathology of retinoblastoma. Am Ophthalmol 1966; 61: 508-14.

7 Parkhill EM, Benedict WL. Gliomas of the retina: a histologic study. Am F Ophthalmol 1941; 24: 1354-73.

8 Tso $M$, Zimmerman LE, Fine BS. The nature of retinoblastoma: I. Photoreceptor differentiation: a clinical and histologic study. Am $\mathcal{F}$ Ophthalmol 1970; 69: 339-50. 